\title{
Une nouvelle myopathie due au déficit de la protéine membranaire lysosomale LAMP-2
}

\begin{abstract}
Les lysosomes sont des vacuoles intracytoplasmiques à $\mathrm{pH}$ acide, riches en hydrolases capables de dégrader la plupart des macromolécules biologiques. Ces enzymes de type lipases, glycosidases, protéases, etc. ont été bien caractérisées au cours des vingt dernières années, mais il est vraisemblable que certaines d'entre elles restent encore à découvrir. La plupart des enzymes connues à ce jour ont pu chacune être associées à une pathologie humaine. Ainsi, plusieurs types de maladies ont été décrits: mucopolysaccharidoses, neurolipidoses, glycoprotéinoses, glycogénose de type II, formant le groupe des maladies lysosomales. Depuis peu, on a également rattaché à cet ensemble les céroïde lipofuscinoses, maladies neurodégénératives impliquant vraisemblablement un grand nombre de gènes (huit suspectés à I'heure actuelle) et venant enrichir un groupe qui atteint d'ores et déjà une cinquantaine d'entités ([1] et $\mathrm{m} / \mathrm{s}$ 1999, n 10, p. 1179).
\end{abstract}

Les lysosomes sont circonscrits par une membrane qui joue en premier lieu un rôle physique en isolant les hydrolases acides des autres composants cytoplasmiques. Par ailleurs, cette membrane est vraisemblablement impliquée dans le maintien de l'environnement acide intralysosomal, dans le transport des produits de dégradation vers le cytoplasme, dans des interactions avec les membranes des autres organites intracellulaires... Contrairement aux enzymes lysosomales, les composants de la membrane du lysosome sont moins bien connus. Cependant, certains d'entre eux ont été caractérisés, telles les molécules LAMP-1(lysosome associated membrane protein-1) et LAMP-2 [2]. II s'agit de glycoprotéines, composées d'un grand domaine situé dans la lumière du branaire et d'une courte queue cytoplasmique. Les protéines LAMP-1 et LAMP-2 sont codées par des gènes différents, localisés respectivement en $13 q 34$ et Xq24-25. De par leur abondance (elles constituent $50 \%$ des protéines membranaires lysosomales), elles forment un manteau continu à la surface interne de la membrane Iysosomale, résistant aux enzymes hydrolytiques grâce à des carbohydrates complexes.

Afin d'analyser la fonction de ces protéines, des modèles animaux ont été créés par invalidation des gènes murins correspondants. Ainsi, des souris déficientes en protéine LAMP1 ont été obtenues par l'équipe de Paul Saftig en 1999 [3]. Ces souris ayant subi une invalidation classique par insertion d'une cassette néo codant pour le phénotype de résistance à la néomycine dans l'exon 3 du gène lamp-1 ne présentent pas de phénotype particulier. Elles sont viables et fertiles. Les analyses histologiques et ultrastructurales des différents tissus ne révèlent pas d'anomalies. Les propriétés des lysosomes sont normales: activités enzymatiques, pH, densité, distribution subcellulaire. Contrairement à ce que I'on pouvait attendre compte tenu de I'abondance de LAMP-1, son déficit est bien toléré chez la souris. Ceci semble s'expliquer par une augmentation compensatrice de l'expression de la protéine LAMP-2, par le biais d'un mécanisme de régulation traductionnelle. II est cependant à noter que les souris déficientes en LAMP-1 présentent au niveau du cerveau une astrogliose modérée, ainsi qu'une distribution anormale de la cathepsine D. Un suivi à plus long terme devrait permettre de déterminer les répercussions de ces signes sur le phénotype des animaux. Tout récemment, un modèle animal déficient en protéine LAMP-2 vient d'être obtenu par la même équipe par invalidation du gène lamp-2 murin [4]. Environ $50 \%$ de ces souris meurent entre 20 et 40 jours de vie, ceci indépendamment du sexe et du fond génétique. Ces souris présentent généralement des sténoses ou des infarctus segmentaires de l'intestin grêle, ainsi que des lésions pancréatiques. Celles qui survivent sont fertiles, mais sont de petite taille et présentent une cardiomyopathie. L'analyse ultrastructurale des tissus montre une accumulation massive de vacuoles autophagiques dans le foie, le pancréas, la rate, le rein, ainsi que dans le muscle squelettique et cardiaque.

L'équivalent humain de ce déficit en protéine LAMP-2 vient également d'être caractérisé [5]. II s'agit de la maladie de Danon, décrite pour la première fois en 1981 chez deux garçons présentant une cardiomyopathie associée à une myopathie et à un retard mental [6]. Cette maladie possède des caractéristiques cliniques et ultrastructurales proches de la maladie de Pompe, une glycogénose d'origine lysosomale, due au déficit de l' $\alpha$ glucosidase ou maltase acide [7]. La maladie de Danon a d'ailleurs été décrite comme une "glycogénose Iysosomale avec maltase acide normale». Depuis la description originale, plusieurs patients ont été décrits, présentant le même tableau clinique avec une myopathie vacuolaire caractéristique à la biopsie musculaire. Les vacuoles intra-cytoplasmiques retrouvées dans le muscle squelettique et cardiaque sont limitées par une membrane et contiennent du matériel autophagique et du glycogène.

$L$ 'analyse du gène LAM P-2 chez dix patients, incluant ceux décrits initialement par Danon a permis de mettre en évidence huit mutations différentes, à type de délétions ou d'insertions de 2 à 10 bases, de mutations non-sens ou d'anomalies d'épis- 
sage. Il est donc vraisemblable que la maladie de Danon soit due à un déficit primaire en protéine LAMP-2, dont il reste à définir le rôle exact.

Cette maladie a un mode de transmission récessif lié au sexe chez la souris et chez l'homme, les gènes lamp-2 humain et murin étant situés sur le chromosome $X$. Cependant, il est clair que les «glycogénoses lysosomales avec maltase acide normale» ne sont pas une seule et même maladie, puisqu'on connaît des formes à transmission dominante [8]. La suite du travail devrait permettre de définir les différents variants phénotypiques de la maladie de Danon et de rechercher les gènes responsables des autres affections de ce groupe. Par ailleurs, la maladie de Danon est le premier exemple de myopathie humaine due à des anomalies d'une protéine lysosomale membranaire. Des maladies impliquant d'autres pro- téines de ce type sont connues, telles la cystinose [9] ou la maladie de Salla [10], affectant des transporteurs, respectivement de la cystine et de l'acide sialique. II est clair que la saga des protéines membranaires lysosomales ne fait que commencer et que leur pathologie va certainement, dans les années à venir, renforcer le groupe déjà vaste des maladies lysosomales.

1. Neufeld EF. Lysosomal storage diseases. Annu Rev Biochem 1991; 60: 257-80.

2. Fukuda M. Lysosomal membrane glycoproteins. Structure, biosynthesis, and intracellular trafficking. J Biol Chem 1991; 266: 21327-30.

3. Andrejewski N, Punnonen EL, Guhde G, et al. Normal lysosomal morphology and function in LAMP-1-deficient mice. J Biol Chem 1999; 274: 12692-701.

4. Tanaka Y, Guhde G, Suter A, et al. Accumulation of autophagic vacuoles and cardiomyopathy in LAMP-2-deficient mice. Nature 2000; 406: 902-6.

5. Nishino I, Fu J, Tanji K, et al. Primary LAMP-2 deficiency causes vacuolar cardiomyopathy and myopathy (Danon disease). Nature 2000; 406: 906-10.
6. Danon MJ, Oh SJ, DiMauro S et al. Lysosomal glycogen storage disease with normal acid maltase. N eurology $1981 ; 31: 51-7$.

7. H irschhorn R. Glycogen storage disease type II: acid $\alpha$-glucosidase (acid maltase) deficiency. In: Scriver CR, Beaudet AL, Sly WS, Valle D, eds. The metabolic and molecular bases of inherited disease. NewYork: Mc Graw Hill, 1995: 2443-64.

8. Byrne E, Dennett X, Crotty B, et al. Dominantly inherited cardioskeletal myopathy with Iysosomal glycogen storage and normal acid maltase levels. Brain 1986; 109: 523-36.

9. Town $M$, Jean $G$, Cherqui $S$, et al. A novel gene encoding an integral membrane protein is mutated in nephropathic cystinosis. Nat Genet 1998; 18: 319-24.

10. Verheijen FW, Verbeek $E$, Aula $N$, et al. A new gene, encoding an anion transporter, is mutated in sialic acid storage diseases. Nat Genet 1999; 23: 462-5.

\section{Catherine Caillaud \\ Livia Poenaru}

Laboratoire de Génétique et Inserm U. 129, CHU Cochin-Port-Royal, 24, rue du Faubourg-Saint-Jacques, 75014 Paris, France.

\section{BRÈVES}

À l'écoute des moteurs dans les surdités. Les myosines sont pourvues d'une tête qui glisse le long des filaments d'actine. Ce sont de véritables moteurs moléculaires, mais leurs différentes fonctions sont loin d'être complètement élucidées [1]. Les myosines de classe II (ou conventionnelles) sont largement exprimées dans les muscles squelettiques, cardiaques, lisses et dans de nombreux autres tissus. Elles sont constituées d'une paire de chaînes lourdes, d'une paire de chaînes légères et d'une paire de chaînes légères régulatrices [2]. La connaissance du rôle biologique des myosines non musculaires provient en partie des études faites chez les invertébrés et des analogies avec la myosine II des muscles lisses et des muscles squelettiques. En ce qui concerne les surdités, I'implication de trois myosines a été démontrée (m/s 1995, $n^{\circ} 8$, p. 1181) et [2] : les myosines VI, VIIA et XVII qui sont non conventionnelles (c'est-à-dire n'appartenant pas à la classe II) . Pour la première fois, une myosine conventionnelle vient d'être impliquée dans une surdité. Le gène
M YH 9, codant pour une chaîne lourde de myosine humaine non musculaire de type $A$, et localisé en 22q11.2 est en effet muté dans une surdité dominante autosomique non syndromique peu fréquente, DFNA17, [3]. La mutation observée touche une région très conservée dans l'évolution qui doit altérer I'hélice $\mathrm{SH} 1$. Or, on sait que les hélices $\mathrm{SH} 1$ et $\mathrm{SH} 2$ jouent un rôle essentiel dans les changements de conformation du domaine moteur au niveau de la tête de la myosine. Dans la DFNA17, I'atteinte auditive débute vers l'âge de 10 ans, touche surtout les hautes fréquences et, à partir de la troisième décennie, entraîne une surdité plus ou moins sévère qui ressemble à la presbyacousie de la sénescence. L'étude histopathologique chez un malade montre une dysplasie cochléosacculaire avec dégénérescence de l'organe de Corti, de l'épithélium sacculaire et de la strie vasculaire [4]. Chez le rat, l'immunolocalisation de MYH 9 montre son expression dans trois parties distinctes de la cochlée: l'organe de Corti au sein des cellules ciliées externes (m/s 2000, $n^{\circ} 2$, p. 270), la région subcentrale du ligament spiral et la membrane de Reissner*. Or, chez ce même malade, la membrane de Reissner était effondrée. On peut donc se demander si l'anomalie de la membrane de Reissner entraîne un trouble de l'homéostasie, ou si l'atteinte des fibrocytes du ligament spiral (en particulier fibrocytes III riches en actine) provoque des troubles de la tension du complexe ligament spiral-membrane basilaire, tension qui doit être importante pour la sensibilité aux hautes fréquences dans le tour basal de la cochlée. Mais nous n'en sommes encore qu'à des conjectures.

[1. Probst FJ, et al. Science 1998; 280: 1444-7.]

[2. Sellers ]R. Biochim Biophys Acta 2000; 1496: 3-22.]

[3. Lalwani AK, et al. Am J Hum Genet 2000; 67: 1121-8.]

[4. Lalwani AK, et al. Audiol Neurootol $1997 ; 2$ : 139-54.]

* Structure constitué de cellules épithéliales unies par des jonctions serrées reposant sur une couche mésothéliale lâche en regard de la rampe périlymphatique vestibulaire 